\title{
The birth of the Malaysian society for music in medicine: a concerted move to promote the use of music for therapeutic purposes
}

\begin{abstract}
Malaysia is a multi-cultural society and the resulting myriad forms of music played in the nation are an ethnomusicologist's haven. A simple cross-sample taken across the different kinds of music available reflects a diverse range reflective of much of the Asian region as a whole. Music medicine has existed for as long as the indigenous tribes have lived in the region. Formalized music therapy started in Malaysia approximately two decades ago by way of Western trained music therapists and is still in its infancy in therapeutic usage. As allopathic practitioners increasingly develop an evidence-based holistic mindset to complementary healing modalities, it is hoped that the use of music for therapeutic purposes will increase over time, and be localized for a population that blends Western, Chinese, Indian and indigenous healing systems. Efforts are made to conduct research using musicbased intervention to provide evidence for integration of music medicine into Malaysian healthcare.
\end{abstract}

Keyword: Analgesic consumption; Malaysia; Music medicine; Music therapy; Palliative 